\title{
Associated Risk Factors in Pre-diabetes and Type 2 Diabetes in Saudi Community
}

\author{
Khalid S Aljabri ${ }^{1 *}$, Samia A Bokhari ${ }^{1}$, Muneera A Alshareef ${ }^{1}$, Patan M Khan ${ }^{1}$ and Bandari K Aljabri ${ }^{2}$ \\ ${ }^{1}$ Department of Endocrinology, King Fahad Armed Forces Hospital, Kingdom of Saudi Arabia \\ ${ }^{2}$ College of medicine, Um Al Qura University, Kingdom of Saudi Arabia
}

Received: 眥 May 29, 2018; Published: 制 June 11, 2018

*Corresponding author: Khalid S.J. Aljabri, Department of Endocrinology, King Fahad Armed Forces Hospital, P0 Box 9862, Jeddah 21159, Kingdom of Saudi Arabia

\begin{abstract}
Background and Objective: The prevalence and incidence of type 2 diabetes mellitus

(T2DM) are increasing worldwide. Pre diabetes is a high-risk state for the development of diabetes and its associated complications. This study aims to determine the associated risk factors among T2DM and pre diabetes patients among adult Saudi population.
\end{abstract}

Methods: For the present study, we analyzed participants who are older than 20 years old

and had undergone a blood test to assess HbA1c. A total of 1095 were selected to be enrolled for the present study. All patients were from the population of the Primary health and Diabetic Centres at King Fahad Armed Forces Hospital. Participants were defined as having T2DM according to self-report, clinical reports, use of anti diabetic agents and HbA1c ( 26.5$)$. Non T2DM participants were divided into normoglycemic or pre diabetic group as follows: HbA1c < 5.7, (normoglycemic) or HbA1c 5.7-6.4 (pre diabetes). Laboratory assessments included HbA1c, lipids, creatinine and urinary micro albumin.

Main results: Of the 1095 participants analyzed, 796 were women (72.7\%). Age was $45.1 \pm 11.1$ and BMI was $30.7 \pm 5.7$. Hypertension had been diagnosed in 415 (38.2\%) participants. Blood measurements revealed the following values: creatinine $68.2 \pm 22.0 \mathrm{umol} / \mathrm{L}$, Urine micro albumin (g/min) 55.4 \pm 200.3 , total cholesterol levels $4.9 \pm 1.0 \mathrm{mmol} / \mathrm{L}$, high density lipoprotein $1.3 \pm 0.3 \mathrm{mmol} / \mathrm{L}$, triglyceride levels $1.5 \pm 0.7$ and low density lipoprotein $3.0 \pm 0.9 \mathrm{mmol} / \mathrm{L}$. Of the overall 1095 analyzed participants, pre diabetes was present in 362(33.1\%), 368(33.6\%) were classified as T2DM and 365 (33.3\%) as normoglycemic. When comparing pre diabetic with normoglycemic and T2DM population, pre diabetic subjects were more likely to have hypertension and higher triglyceride than normoglycemic but less than T2DM subjects. In addition, pre diabetic patients compared with T2DM ones had higher levels of low density lipoprotein and high density lipoprotein. Logistic regression analysis showed no significant association of any of the co variables with normoglycemic subjects in front of the pre diabetic reference group, whereas the odds of being in the diabetic group gets multiplied by 7.56 for each unitary increase in male gender ( $\mathrm{p}<0.0001$, OR: 7.56, 95\% CI 3.16-18.23). Also, individuals with hypertension had higher odds of being in the DM group than in the prediabetic ( $\mathrm{p}<0.0001,0 \mathrm{R}: 6.06,95 \%$ CI 3.2511.28). Age of subjects had lower odds of being in the DM group than in the pre diabetic ( $\mathrm{p}<0.0001,0 \mathrm{R}: 0.85,95 \%$ CI $(0.82-0.89)$.

Conclusion: This study found the major clinical differences between pre diabetic and T2DM Patients were the higher hypertension and hypertriglyceridenia in the T2DM patients. Clearly, despite the small sample size, this study has posed important public health issues that require immediate attention from the health authority. Unless immediate steps are taken to contain the increasing prevalence of obesity, diabetes, pre diabetes, the health care costs for chronic diseases will pose an enormous financial burden to the country.

Keywords: Type 2 Diabetes; Pre diabetes; Risk factors

Abbreviations: T2DM: Type 2 Diabetes Mellitus; IFG: Impaired Fasting Glucose; BMI: Body Mass Index; HTN: Hypertension; AER: Albumin Excretion Rate; DN: Diabetic Nephropathy; OR: Odds Ratio; CI: Confidence Interval; I-IFG: Isolated Impaired Fasting Glucose 


\section{Introduction}

Diabetes mellitus is a major cause of excess mortality and morbidity. The prevalence and incidence of type 2 diabetes mellitus (T2DM) are increasing worldwide [1]. T2DM patients have a higher risk of developing microvascular and macrovascular disease than the general population. The occurrence of these complications depends largely on the degree of glycemic control as well as on the adequate control of cardiovascular risk factors [2-5]. In Saudi Arabia, primary epidemiological diabetes features are not different. The diabetes mellitus prevalence among adult Saudi population has reached $23.7 \%$, a percentage being the highest across the globe $[6,7]$. Statistics regarding the increasing trend of diabetes and pre diabetes in the world have also been observed in Saudi Arabia. As per the WHO country profile 2016, 14.4\% of Saudi population has diabetes, while prevalence in males is $14.7 \%$ [8]. In 2015, the prevalence of pre diabetics was found to be $9.0 \%$ in Jeddah with $9.4 \%$ in men, while for diabetes, it was $12.1 \%$ with $12.9 \%$ adult male population suffering from it [9]. Another study conducted in Saudi population revealed that the diabetes prevalence in their study was found to be $25.4 \%$, while impaired fasting glucose (IFG) was $25.5 \%$. The strongest risk factors were age $>45$ years, high triglycerides levels, and hypertension [10].

Pre diabetes is a high-risk state for the development of diabetes and its associated complications [11-13].

Recent data have shown that in developed countries, such as the Unites States and the United Kingdom, more than one-third of adults have pre diabetes, but most of these individuals are unaware they have the condition [14-16]. Once detected, pre diabetes needs to be acknowledged with a treatment plan to prevent or slow the transition to diabetic $[17,18]$. Treatment of pre diabetes is associated with delay of the onset of diabetes [19]. Detection and treatment of pre diabetes is therefore a fundamental strategy in diabetes prevention [11].

Current recommendations for pre diabetes screening by the American Diabetes Association focus nearly exclusively on adults who are overweight or obese as defined by body mass index (BMI) until the patient meets the age-oriented screening at 45 years [11]. Further, the recently released recommendation from the US Preventive Services Task Force regarding screening for abnormal glucose levels and T2DM limits screening to individuals who are overweight or obese [20]. This focus on obese or overweight individuals, although obesity and pre diabetes have shown trends of increasing prevalence. United States Preventive Services Task Force has recommended screening of diabetes in adults devoid of precise symptoms and in individuals with BP higher than 135/80mmHg [21]. This study aims to determine the associated risk factors among T2DM and pre diabetes patients among adult Saudi population.

\section{Methods}

For the present study, we analyzed participants who are older than 20 years old and had undergone a blood test to assess HbA1c. A total of 1095 were selected to be enrolled for the present study. All patients were from the population of the Primary health and Diabetic Centers at King Fahad Armed Forces Hospital. Participants were defined as having T2DM according to self-report, clinical reports, use of anti diabetic agents and HbA1c ( $\geq 6.5)$ [11]. Non T2DM participants were divided into normoglycemic or pre diabetic group as follows: HbA1c $<5.7$, (normoglycemic) or HbA1c 5.7-6.4 (pre diabetes) [11]. 362 subjects were found to be pre diabetic. Almost similar number of normoglyceic and T2DM subjects was selected to be analyzed for comparison. All data were collected by personal interview and on the basis of a review of electronic medical data. Weight $(\mathrm{kg})$ and height $(\mathrm{cm})$ were measured by physician and nurse interviewers and recorded. Overweight and obesity were defined as BMI $25-29.9$ and $\geq 30.0 \mathrm{~kg} / \mathrm{m} 2$ respectively [22]. Blood Pressure readings were within a gap of 15 minutes using a mercury sphygmomanometer by palpation and auscultation method in right arm in sitting position. Two readings were taken $15 \mathrm{~min}$ apart and the average of both the readings was taken for analysis. Hypertension (HTN) was also diagnosed based on anti HTN medications or having a prescription of antihypertensive drugs and were classified as Hypertensive irrespective of their current blood pressure reading or if the blood pressure was greater than 140/90 mmHg i.e. systolic BP more than 140 and diastolic BP more than 90 $\mathrm{mm}$ of $\mathrm{Hg}$ - Report of the American College of Cardiology/American Heart Association Task Force on Clinical Practice Guidelines [23]. Laboratory assessments included HbA1c, lipids, creatinine and urinary micro albumin. HbA1c was expressed as percentage. High performance liquid chromatography was used. Fasting serum lipids were measured on a sample of blood after fasting for 14 hours. We used the enzymatic method for determining the cholesterol and trigylcerides levels. Diabetic nephropathy (DN) was assessed by measurement of mean albumin excretion rate (AER) on timed, overnight urine collections. We use a polyclonal radioimmunoassay for albumin measurement. DN is defined as an albumin excretion rate of $>20 \mathrm{~g} / \mathrm{min}$ in a timed or a $24 \mathrm{hr}$ urine collection which is an equivalent to $>30 \mathrm{mg} / \mathrm{g}$ creatinine in a random spot sample.

\section{Statistical Analysis}

Univariate analysis of demographic and clinical laboratory was accomplished using one-way analysis of variance (ANOVA) with posy hoc analysis between variables, to estimate the significance of different between groups where appropriate. Chi square (X2) test were used for categorical data comparison. The adjusted odds ratio (OR) with a 95\% confidence interval (CI) was calculated. In order to evaluate the adjusted association of aforementioned factors on being normoglycemic or diabetic in relation to the pre diabetes group, a multinomial logistic regression model was fit, in which the categorical dependent variable was normoglycemia, pre diabetes or 
T2DM(with pre diabetes as the reference category), and significant variables in bivariate analyses were included as explanatory variables. Despite of the ordinal nature of the dependent variable, ordered logistic regression was not adjusted because the aim of the study was not the association of factors with a latent degree of diabetes but the differential profile of pre diabetes in front of normoglicemia and diabetes. As all the participants were the same age, adjusting for age was not applied. All statistical analyses were performed using SPSS Version 22.0. The difference between groups was considered significant when $\mathrm{P}<0.05$.

\section{Results}

Of the 1095 participants analyzed, 796 were women (72.7\%). Age was $45.1 \pm 11.1$ and BMI was $30.7 \pm 5.7$. Hypertension had been diagnosed in 415 (38.2\%) participants. Blood measurements revealed the following values: creatinine $68.2 \pm 22.0 \mathrm{umol} / \mathrm{L}$, Urine microalbumin (g/min) 55.4 \pm 200.3 , total cholesterol levels $4.9 \pm 1.0 \mathrm{mmol} / \mathrm{L}$, high density lipoprotein $1.3 \pm 0.3 \mathrm{mmol} / \mathrm{L}$, triglyceride levels $1.5 \pm 0.7$ and low density lipoprotein 3.0 $\pm 0.9 \mathrm{mmol} / \mathrm{L}$. Of the overall 1095 analyzed participants, pre diabetes was present in 362(33.1\%), 368(33.6\%) were classified as T2DM and $365(33.3 \%)$ as normoglycemic. Table 1 shows the clinical characteristics and laboratory data of the three groups according to the predefined glycemic status. When comparing pre diabetic with normoglycemic and T2DM population, pre diabetic subjects were more likely to have hypertension and higher triglyceride than normoglycemic but less than T2DM subjects. In addition, prediabetic patients compared with T2DM ones had higher levels of low density lipoprotein and high density lipoprotein. In Table 2, logistic regression analysis showed no significant association of any of the covariables with normoglycemic subjects in front of the pre diabetic reference group, whereas the odds of being in the diabetic group gets multiplied by 7.56 for each unitary increase in male gender ( $\mathrm{p}<0.0001$, OR: 7.56, 95\% CI 3.16-18.23). Also, individuals with hypertension had higher odds of being in the DM group than in the pre diabetic ( $p<0.0001$, OR: 6.06, 95\% CI 3.25-11.28). Age of subjects had lower odds of being in the DM group than in the pre diabetic ( $\mathrm{p}<0.0001$, OR: 0.85, 95\% CI (0.82-0.89).

Table 1: Characteristics of patients with Normoglycemia, prediabetes and type 2 diabetes mellitus.

\begin{tabular}{|c|c|c|c|c|c|c|c|c|}
\hline \multirow{2}{*}{\multicolumn{2}{|c|}{ Parameters }} & \multirow[b]{2}{*}{ Normolglycemia } & \multirow[b]{2}{*}{ Prediabetes } & \multirow[b]{2}{*}{ T2DM } & \multicolumn{4}{|c|}{$P$ value } \\
\hline & & & & & Total & $\begin{array}{l}\text { Normolglycemia } \\
\text { vs Prediabetes }\end{array}$ & $\begin{array}{l}\text { Normolglycemia } \\
\text { vs T2DM }\end{array}$ & $\begin{array}{c}\text { Prediabetes } \\
\text { vs T2DM }\end{array}$ \\
\hline \multicolumn{2}{|c|}{ Number (\%) } & $365(33.3)$ & $362(33.1)$ & 368 (33.6) & & & & \\
\hline \multicolumn{2}{|c|}{ Age (years) } & $44.1 \pm 13.8$ & $45.9 \pm 13.1$ & $45.4 \pm 2.6$ & 0.08 & 0.03 & 0.1 & 0.5 \\
\hline \multirow{2}{*}{ Gender } & Male & $50(16.7)$ & $64(21.4)$ & $185(61.9)$ & $<0.0001$ & 0.1 & $<0.0001$ & $<0.0001$ \\
\hline & Female & $315(39.6)$ & $298(37.4)$ & $183(23.0)$ & & & & \\
\hline \multicolumn{2}{|c|}{ Hypertension } & $66(15.9)$ & $87(21.0)$ & $262(63.1)$ & $<0.0001$ & 0.04 & $<0.0001$ & $<0.0001$ \\
\hline \multirow{4}{*}{$\begin{array}{l}\text { Body mass index } \\
\left(\mathrm{kg} / \mathrm{m}^{2}\right)\end{array}$} & Mean \pm SD & $30.0 \pm 5.5$ & $31.0 \pm 6.2$ & $31.0 \pm 5.3$ & 0.03 & 0.03 & 0.02 & 0.8 \\
\hline & $<25.0$ & $72(39.8)$ & $63(34.8)$ & $46(25.4)$ & 0.3 & 0.7 & 0.3 & 0.07 \\
\hline & $25.0-29.9$ & $97(32.1)$ & $100(33.1)$ & $105(34.8)$ & & & & \\
\hline & $\geq 30.0$ & $194(34.0)$ & $193(33.8)$ & $184(32.2)$ & & & & \\
\hline \multicolumn{2}{|c|}{ HbA1c } & $5.2 \pm 0.4$ & $6.0 \pm 0.2$ & $8.1 \pm 2.2$ & $<0.0001$ & $<0.0001$ & $<0.0001$ & $<0.0001$ \\
\hline \multicolumn{2}{|c|}{ Total cholesterol (mmol/l) } & $4.8 \pm 1.0$ & $4.9 \pm 0.9$ & $4.9 \pm 1.1$ & 0.4 & 0.3 & 0.2 & 0.8 \\
\hline \multicolumn{2}{|c|}{ Low density lipoprotein (mmol/l) } & $3.0 \pm 0.9$ & $3.0 \pm 0.8$ & $2.9 \pm 0.9$ & 0.5 & 0.95 & 0.3 & 0.3 \\
\hline \multicolumn{2}{|c|}{ Triglyceride (mmol/l) } & $1.3 \pm 0.7$ & $1.4 \pm 0.7$ & $1.7 \pm 0.8$ & $<0.0001$ & 0.05 & $<0.0001$ & $<0.0001$ \\
\hline \multicolumn{2}{|c|}{$\begin{array}{l}\text { High density lipoprotein } \\
(\mathrm{mmol} / \mathrm{l})\end{array}$} & $1.3 \pm 0.3$ & $1.3 \pm 0.3$ & $1.2 \pm 0.3$ & 0.007 & 0.5 & 0.003 & 0.02 \\
\hline \multicolumn{2}{|c|}{ Renal failure } & $9(18.4)$ & $10(20.4)$ & $30(61.2)$ & $<0.0001$ & 0.8 & 0.001 & 0.002 \\
\hline \multicolumn{2}{|c|}{ Diabetic nephropathy } & $14(8.6)$ & $26(16.0)$ & $122(75.3)$ & 0.001 & 0.2 & 0.001 & 0.02 \\
\hline
\end{tabular}

Data are means \pm SD or number $(\%)$

Table 2: Multinomial logistic regression results according to glycemic status. Association with clinical characteristics among prediabetic as a reference category.

\begin{tabular}{|c|c|c|c|c|}
\hline \multirow{2}{*}{ Parameters } & \multicolumn{2}{|c|}{ Normolglycemia } & \multicolumn{2}{c|}{ Type 2 diabetes } \\
\cline { 2 - 5 } & OR (95\% CI) & P value & OR (95\% CI) & P value \\
\hline Age (years) & $1.00(0.98-1.04)$ & 0.7 & $0.85(0.82-0.89)$ & $<0.0001$ \\
\hline Gender (female) & $0.91(0.32-3.56)$ & 0.9 & $7.59(3.16-18.23)$ & $<0.0001$ \\
\hline Hyperetnsion & $0.95(0.50-1.82)$ & 0.9 & $6.06(3.25-11.28)$ & $<0.0001$ \\
\hline Body mass index $\left(\mathrm{kg} / \mathrm{m}^{2}\right)$ & $1.00(0.95-1.06)$ & 0.9 & $1.01(0.96-1.06)$ & 0.8 \\
\hline
\end{tabular}




\begin{tabular}{|c|c|c|c|c|}
\hline Total cholesterol (mmol/l) & $1.14(0.64-2.03)$ & 0.7 & $1.17(0.71-1.95)$ & 0.5 \\
\hline $\begin{array}{c}\text { Low density lipoprotein } \\
\text { (mmol/l) }\end{array}$ & $0.93(0.53-1.65)$ & 0.8 & $0.82(0.50-1.36)$ & 0.4 \\
\hline Triglyceride (mmol/l) & $1.09(0.60-2.00)$ & 0.8 & $1.44(0.86-2.40)$ & 0.2 \\
\hline $\begin{array}{c}\text { High density lipoprotein } \\
\text { (mmol/l) }\end{array}$ & $1.28(0.41-3.98)$ & 0.7 & $1.73(0.59-5.09)$ & 0.2 \\
\hline Renal failure & $5.64(0.56-56.66)$ & 0.1 & $2.66(0.31-22.86)$ & 0.4 \\
\hline Diabetic nephropathy & $0.67(0.29-1.55)$ & 0.35 & $1.22(0.62-2.39)$ & 0.6 \\
\hline
\end{tabular}

\section{Discussion}

This study showed that multiple risk factors are related to T2DM, but not to the pre diabetes group, including age, female gender and HTN. Generalization to all population could not be due to regionalized characteristics. In addition, it does not evaluate the healthcare services offered in our city. The size of our sample and the cross section type of the study should be of consideration.

T2DM is a major health concern worldwide and is increasing in parallel with the obesity epidemic [24]. Prevalence of T2DM has increased dramatically with 1 million people reported to have been diagnosed with T2DM in 1994, increasing to 382 million by 2013, and with prediction of 592 million by 2035 [25]. Given that both genetic and environmental factors contribute to T2DM progression, it has been proposed that amongst increasing globalization, Asian ethnicities including Saudi Arabia have been unable to adapt to food and lifestyle related aspects of westernized culture [26]. Hence when matched for the same gender, age, and body weight, those with Asian ethnicity appear to have a greater risk of poor metabolic health than Caucasian counterparts including Europeans people [27]. This increased risk for T2DM has been reported in both Asians and Saudi Arabia [6-10,28].

Currently, the population with pre-diabetes has reached approximately 318 million around the world, accounting for $6.7 \%$ of the total number of adults. About $69.2 \%$ of the prediabetes population lives in low or middle-income countries [29]. Understanding pre diabetes may be crucial to reducing the global T2DM epidemic and is defined either by the presence of isolated impaired fasting glucose (I-IFG); or isolated impaired glucose tolerance (I-IGT); or both IFG and IGT. To maintain glucose homeostasis greater secretion of insulin is required from the pancreatic cells, and hence hyperinsulinemia develops. Prolonged hyperinsulinemia and/or fatty pancreas may in turn lead to the dysfunction of pancreatic cells, resulting in impaired insulin secretion [30]. Decreased insulin secretion and concomitant increased blood glucose levels consequently also lead to the reduced uptake of glucose by skeletal muscle, thereby enhancing muscle insulin resistance [31]. IFG, determined from fasting plasma glucose, occurs as a result of poor glucose regulation, resulting in raised blood glucose even after an overnight fast, while IGT is due to an individual being unable to respond to glucose consumed as part of a meal, resulting in increased postprandial blood glucose
[11]. More recently, prediabetes has also been identified by mildly elevated HbA1c [32,33].

The younger age of T2DM in our cohort is consistent with that seen among other groups such as the Australians, the American Indian and Alaska natives [34-36]. Age of subjects had lower odds of being in the DM group than in the pre diabetic ( $\mathrm{p}<0.0001,0 \mathrm{R}: 0.85$, 95\% CI (0.82-0.89) in concordance with earlier reports $[37,38]$. Odds of being in the diabetic group gets multiplied by 7.56 for each unitary increase in male gender ( $\mathrm{p}<0.0001$, OR: 7.56, 95\% CI 3.1618.23). As seen in this study, majority of the female participants were either overweight $(59.6 \%)$ or obese $(78.6 \%)$. The reason for such an observation has not been completely elucidated but is proposed to be associated with obesity which is highly prevalent in the populations worldwide. Since obesity is closely linked to increased insulin resistance and decreased insulin sensitivity and higher risk of diabetes, arresting the obesity pandemic among our population should be a priority [39-41]. Special, culturally oriented community-based intervention programs need to be implemented. The frequency of pre diabetes in $27.2 \%$ of the female cases out of the total cohort in this study was six times higher than other, estimated to be $4.2 \%$ in 2006 [42,43]. Due to our small sample size, this is inconclusive and needs to be verified by extending our study to more of our communities. Nevertheless, our findings warrant special attention from the health authorities since although HbA1c is not as sensitive as IGT test, it has consistently been shown to be a good predictor of increased risk for cardiovascular diseases and T2DM in many populations around the world $[44,45]$.

Previous cross-sectional studies have reported that multiple risk factors are related to pre-diabetes, Such as increased age, overweight, obesity, blood pressure, and dyslipidemia [37,46,47]. More importantly, impaired glucose tolerance was found to be an independent risk factor for cardiovascular disease, the hazard ratio of death was 2.22 (95\% CI = 1.08-4.58), and arterial stiffness and pathological changes in the arterial intima occurred in the stage of IGT [48]. The participants in our study with pre-diabetes had higher BMI, more frequent HTN, higher triglyceride, frequent renal failure and DN than those without pre-diabetes but lower than participants with T2DM. logistic regression analysis showed no significant association of any of the covariables with normoglycemic subjects in front of the pre diabetic reference group, whereas the odds of being in the diabetic group gets multiplied by 7.56 for each unitary increase in male gender. Also, individuals with hypertension had 
higher odds of being in the DM group than in the pre diabetic. Age of subjects had lower odds of being in the DM group than in the pre diabetic which was consistent with earlier studies $[37,38]$.

Previous studies have reported that overweight and obesity were the mainly factors contributing to insulin resistance, and insulin resistance was the basis of diabetes and other chronic diseases $[49,50]$. In the present study, BMI was significantly higher in the pre diabetes than the normal groups, $p=0.03$. When BMI was classified into three types. The total numbers of overweight and obese people in the pre-diabetes and normal groups were 293 and 291 , respectively (the total number were 362 and 365, respectively), and there were statistically non significant differences in being overweight or obese between the pre-diabetes and normal groups $(\mathrm{OR}=1.02,95 \% \mathrm{CI}=0.86-1.21, \mathrm{p}=0.8)$. Increasing evidence suggests that the excess body fat in overweight/obese people might lead to increased degradation of fat, which resulted in the production of large amounts of free fatty acids (FFAs). When the level of FFAs was higher in blood, the capacity of liver tissue for insulin-mediated glucose uptake and utilization was lower, so the blood glucose level was high in circulation [51]. In other words, high FFAs in the blood were one of the important pathogenic factors of obesity caused by insulin resistance [52]. The fact that BMI categories was not a significant factor in our study is the cohort mean BMI was in the obesity range, $\mathrm{p}=0.3$. However, the mean BMI was significantly different between the studied groups, $\mathrm{p}=0.03$.

A high level of triglycerides was not significantly associated as a risk factor for developing pre-diabetes and T2DM (OR $=1.09$, $95 \% \mathrm{CI}=(0.60-2.00), \mathrm{P}=0.8,1.44(0.86-2.40), \mathrm{P}=0.2)$ respectively. High level of triglycerides could increase the fat deposition in muscle, liver, and pancreas, and it could damage the function of mitochondria and induce oxidative stress which, in turn, could cause insulin resistance, but also lead to impaired islet B cell function [53]. Some studies suggested an interrelation between hyper triglyceridemia and insulin resistance and that they promote each other's development $[54,55]$. In concordance with our result, in some epidemiological studies, for instance, the Framingham Heart Study, hyper triglyceridemia was more prevalent in type 2 diabetes mellitus patients than in the normal population, suggesting that hyper triglyceridemia is a causal factor of type 2 diabetes mellitus [56]. However, this paper was a cross-sectional study, thus it was impossible to determine the causal relationship between hyper triglyceridemia and pre-diabetes and T2DM.

Hypertension was found to be a risk factor for T2DM but not for the pre diabetes group in our study $(\mathrm{OR}=6.06,95 \% \mathrm{CI}=3.25$ $11.28, \mathrm{p}<0.0001, \mathrm{OR}=0.95,95 \% \mathrm{CI}=0.50-1.82, \mathrm{p}=0.9$ ) respectively. A possible mechanism is that the activity of angiotensin II is increased in the circulatory system of patient with hypertension. Angiotensin II activates renin-angiotensin-aldosterone system and affects the function of the pancreatic islets, resulting in islet fibrosis and reduced synthesis of insulin, and ultimately leading to insulin resistance $[57,58]$. Insulin resistance can also aggravate the condition of hypertension. Directly or indirectly through the activity of renin-angiotensin-aldosterone system, insulin promotes renal tubular to reabsorb $\mathrm{Na}+$ and water, leading to the increased blood volume and cardiac output; this is considered as one of reasons for the development of hypertension [59]. Interactions between abnormal glucose tolerance, hypertension, and dyslipidemia could impair endothelial cell and result in atherosclerosis or other cardiovascular complications. Therefore, the management of daily diet of people with pre-diabetes and the monitoring of body weight, blood lipids, and blood pressure is very important.

Results of our investigation must be interpreted in light of some limitations such as the cross-sectional design, which does not let to establish any causal relation with respect to prediabetic state and only provides mere associations. Moreover, the classification of glycemic state was based on $\mathrm{HbA1c}$, instead of its combination with a glucose tolerance test. Then, it is expected that the lack of glucose tolerance test data leads to a suboptimal estimation of glycemic state because normoglycemic group may include some individuals with impaired glucose tolerance that should have been included in pre diabetic group. Considering the goal population, a larger cohort would have probably provided a greater power of the statistical analyses.

\section{Conclusion}

This study found the major clinical differences between pre diabetic and T2DM patients were the higher hypertension and hyper triglyceridenia in the T2DM patients. Clearly, despite the small sample size, this study has posed important public health issues that require immediate attention from the health authority. Unless immediate steps are taken to contain the increasing prevalence of obesity, diabetes, pre diabetes, the health care costs for chronic diseases will pose an enormous financial burden to the country.

\section{Acknowledgment}

We are grateful to the staffs from the diabetic centre at King Fahad Armed Forces Hospital for their valuable contributions in data collection. The authors have no conflict of interest to disclose.

\section{References}

1. Mata M, Antoñanzas F, Tafalla M, Sanz P (2002) The cost of type 2 diabetes in Spain: The CODE-2 study. Gac Sanit 16(6): 511-520.

2. American Diabetes Association (2002) Implications of the United Kingdom Prospective Diabetes Study. Diabetes Care 25(Suppl. 1): S28-S32.

3. Holman RR, Paul SK, Bethel MA, Matthews DR, Neil HAW (2008) 10-year follow-up of intensive glucose control in type 2 diabetes. $\mathrm{N}$ Engl J Med 359(15): 1577-1589.

4. Gaede P, Lund-Andersen H, Parving HH, Pedersen O (2008) Effect of a multifactorial intervention on mortality in type 2 diabetes. $\mathrm{N}$ Engl J Med 358: 580-591. 
5. UK Prospective Diabetes Study (UKPDS) Group (1998) Intensive blood-glucose control with sulphonylureas or insulin compared with conventional treatment and risk of complications in patients with type 2 diabetes (UKPDS 33). Lancet 352(9131): 837-853.

6. T. Jalboukh (2017) Health report on Saudi Arabia.

7. MM AlNozha, MA Al Maatouq, Y YAl Mazrou, SS Al Harthi, MR Arafah, et al. (2004) Diabetes mellitus in Saudi Arabia. Saudi Medical Journal 25(11): 1603-1610.

8. World Health country Organization (2016) Diabetes profiles, Saudi Arabia.

9. SM Bahijri, HA Jambi, RMAl Raddadi, GFerns, J Tuomilehto (2016) The prevalence of diabetes and prediabetes in the adult population of Jeddah, Saudi Arabia-a community based survey. PLoS One 11(4): e0152559.

10. K Al Rubeaan, HA Al Manaa, TA Khoja, et al. (2015) Epidemiology of abnormal glucose metabolism in a country facing its epidemic: SAUDIDM study. Journal of Diabetes 7(5): 622-632.

11. American Diabetes Association (2018) Classification and Diagnosis of Diabetes: Standards of Medical Care in Diabetes-2018. Diabetes Care 41 (Suppl1): S13-S27.

12. Mainous AG, Tanner RJ, Coates TD, Baker R (2014) Prediabetes, elevated iron and all-cause mortality: a cohort study. BMJ Open 4(12): e006491.

13. Player MS, Diaz VA, Mainous AG, Gregorie SH, Knoll ME, et al. (2011) Ethnic differences in the relationship of prediabetes with the presence of target-organ disease. Diabetes Metab 37(5):403-409.

14. Bullard KM, Saydah SH, Imperatore G, et al. (2013) Secular changes in U.S Prediabetes prevalence defined by hemoglobin A1c and fasting plasma glucose: National Health and Nutrition Examination Surveys,1999-2010. Diabetes Care 36(8): 2286-2293.

15. Mainous AG, Tanner RJ, Baker R, Zayas CE, Harle CA (2014) Prevalence of prediabetes in England from 2003 to 2011: population-based, crosssectional study. BMJ Open 4(6): e005002.

16. Centers for Disease Control and Prevention (CDC) (2013) Awareness of prediabetes-United States, 2005-2010. MMWR Morb Mortal Wkly 62(11): 209-212.

17. Dunkley AJ, Bodicoat DH, Greaves CJ, et al. (2014) Diabetes prevention in the real world: effectiveness of pragmatic lifestyle interventions for the prevention of type 2 diabetes and of the impact of adherence to guideline recommendations: a systematic review and meta analysis. Diabetes Care 37(4):922-933.

18. Knowler WC, Barrett-Connor E, Fowler SE (2002) Diabetes Prevention Program Research Group. Reduction in the incidence of type 2 diabetes with lifestyle intervention or metformin. N Engl J Med 346(6):393-403.

19. Selph S, Dana T, Blazina I, Bougatsos C, Patel H, et al. (2015) Screening for type 2 diabetes mellitus: a systematic review for the U.S. Preventive Services Task Force. Ann Intern Med 162(11):765-766.

20. Final Update Summary (2015) Abnormal Blood Glucose and Type 2 Diabetes Mellitus: Screening. US Preventive Services Task Force.

21. R. Valdez (2009) Detecting undiagnosed type 2 diabetes: family history as a risk factor and screening tool. Journal of Diabetes Science and Technology 3(4):722-726.

22. World Health Organization (1999) Obesity: preventing and managing the global epidemic: report of a WHO consultation.

23. Whelton PK, Carey RM, Aronow WS, Casey DE, Collins KJ (2017) ACC/ AHA/AAPA/ABC/ACPM/AGS/APhA/ASH/ASPC/NMA/PCNA Guideline for the Prevention, Detection, Evaluation, and Management of High Blood Pressure in Adults: A Report of the American College of Cardiology/ American Heart Association Task Force on Clinical Practice Guidelines. J Am Coll Cardiol 71(6): 1269-1324.
24. Alberti KG, Zimmet PZ (2014) Diabetes: A look to the future. Lancet Diabetes Endocrinol 2(1): 1-2.

25. Zimmet PZ, Magliano DJ, Herman WH, Shaw JE (2014) Diabetes: A $21^{\text {st }}$ century challenge. Lancet Diabetes Endocrinol 2(1): 56-64.

26. Akiyama C (2008) Bridging the gap between two cultures: An analysis on identity attitudes and attachment of Asian Americans. Brief Treat Crisis Interv 8(3): 251-263.

27. Haldar S, Chia SC, Henry, CJ (2012) Body composition in Asians and Caucasians: Comparative analyses and influences on cardiometabolic outcomes. Adv Food Nutr 75: 97-154

28. Pandit K, Goswami, S, Ghosh S, Mukhopadhyay P, Chowdhury S (2012) Metabolic syndrome in South Asians. Indian J Endocrinol Metab 16(1): 44-55

29. International Diabetes Federation (2015) IDF Diabetes Atlas $7^{\text {th }}$ Edition

30. Catoi AF, Parvu A, Muresan A, Busetto L (2015) Metabolic mechanisms in obesity and type 2 diabetes: Insights from bariatric/metabolic surgery. Obes Facts 8(6): 350-363.

31. Kahn SE, Hull RL, Utzschneider KM (2006) Mechanisms linking obesity to insulin resistance and type 2 diabetes. Nature 444(7121): 840-846.

32. Colagiuri S (2011) Epidemiology of prediabetes. Med Clin N Am 95(2): 299-307.

33. Ferrannini E (2014) Definition of intervention points in prediabetes. Lancet Diabetes Endocrinol 2(8): 667-675.

34. Maple-Brown L, Cunningham J, Dunne K, Whitbread C, Howard D, et al. (2008) Complications of diabetes in urban Indigenous Australians: The DRUID study. Diabetes Res Clin Pract 80(3): 455-462.

35. Acton KJ, Burrows NR, Moore K, Querec L, Geiss LS, et al. (2002) Trends in diabetes prevalence among American Indian and Alaska native children, adolescents, and young adults. Am J Public Health 92(9):1485-1490.

36. Abate N, Chandalia M (2003) The impact of ethnicity on type 2 diabetes. J Diabetes Complications 17(1):39-58.

37. Li S, Guo S, He F, Zhang M, He J, et al. Prevalence of diabetes mellitus and impaired fasting glucose, associated with risk factors in rural kazakh adults in Xinjiang, China. Int J Environ Res Public Health 12(1): 554-565.

38. Zhao M, Lin H, Yuan Y, et al. (2016) Prevalence of Pre-Diabetes and Its Associated Risk Factors in Rural Areas of Ningbo, China. International Journal of Environmental Research and Public Health 13(8): 808.

39. Must A, Strauss RS (1999) Risks and consequences of childhood and adolescent obesity. Int J Obes Relat Metab Disord 23(Suppl 2): S2-S11.

40. Yu CH, Zinman B (2007) Type 2 diabetes and impaired glucose tolerance in aboriginal populations: A global perspective. Diabetes Res Clin Pract 78(2):159-170

41. Schienkiewitz A, Schulze MB, Hoffmann K, Kroke A, Boeing H (2006) Body mass index history and risk of type 2 diabetes: Results from the European Prospective Investigation into Cancer and Nutrition (EPIC)Potsdam Study. Am J Clin Nutr 84(2):427-433.

42. (1996) National Health Morbidity Survey, Diabetes .Institute for Public Health, Ministry of Health, Malaysia, p. 9

43. Dea K, Cunningham J, Maple-Brown L, Weeramanthri T, Shaw J, et al (2008) Diabetes and cardiovascular risk factors in urban Indigenous adults: Results from the DRUID study. Diabetes Res Clin Pract 80(3):483489.

44. Barzilay JI, Spiekerman CF, Wahl PW, Kuller LH, Cushman M, et al (1999) Cardiovascular disease in older adults with glucose disorders: Comparison of American Diabetes Association criteria for diabetes mellitus with WHO criteria. Lancet 354(9179): 622-625. 
45. Chen KT, Chen CJ, Gregg EW, Imperatore G, Narayan KM (2003) Impaired fasting glucose and risk of diabetes in Taiwan: Follow-up over 3 years. Diabetes Res Clin Pract 60(3):177-182.

46. Pham NM, Eggleston K (2016) Prevalence and determinants of diabetes and prediabetes among vietnamese adults. Diabetes Res Clin Pract 113 116-124.

47. Tian H, Song G, Xie H, Zhang H, Tuomilehto J, et al. (2009) Prevalence of diabetes and impaired fasting glucose among 769,792 rural Chinese adults. Diabetes Res Clin Pract 84(3): 273-278.

48. Tominaga M, Eguchi H, Manaka H, Igarashi K, Kato T, et al. (1999) Impaired glucose tolerance is a risk factor for cardiovascular disease, but not impaired fasting glucose. The Funagata Diabetes Study. Diabetes Care 22(6): 920-924.

49. Jia WP, Wang C, Jiang S, Pan JM (2010) Characteristics of obesity and its related disorders in China. Biomed Environ Sci 23(1): 4-11.

50. Despres JP, Lemieux I (2006) Abdominal obesity and metabolic syndrome. Nature 444(7121): 881-887.

51. Ni Y, Zhao L, Yu H, Ma X, Bao Y, et al. (2015) Circulating unsaturated fatty acids delineate the metabolic status of obese individuals. E Bio Medicine 2(10): 1513-1522.

52. Boden G (1997) Role of fatty acids in the pathogenesis of insulin resistance and NIDDM. Diabetes 46(1): 3-10.
53. Amati F, Dube JJ, Alvarez Carnero E, Edreira MM, Chomentowski P (2011) Skeletal muscle triglycerides, diacylglycerols, and ceramides in insulin resistance: Another paradox in endurance-trained athletes? Diabetes 60(10): 2588-2597.

54. Giannini C, Santoro N, Caprio S, Kim G, Lartaud D (2011) The triglycerideto-HDL cholesterol ratio: Association with insulin resistance in obese youths of different ethnic backgrounds. Diabetes Care 34(8): 1869-1874.

55. Reaven GM (1993) Role of insulin resistance in human disease (syndrome X): An expanded definition. Annu Rev Med 44: 121-131.

56. Wilson PW, Kannel WB, Anderson KM (1985) Lipids, glucose intolerance and vascular disease:

57. The Framingham Study. Monogr Atheroscler 13: 1-11.

58. Ip S.P, Chan, YW, Che CT, Leung PS (2002) Effect of chronic hypoxia on glutathione status and membrane integrity in the pancreas. Pancreatology 2(1): 34-39.

59. Wang XP, Zhang R, Wu K, Wu L, Dong Y (2004) Angiotensin II mediates acinar cell apoptosis during the development of rat pancreatic fibrosis by AT1R. Pancreas 29(4): 264-270.

60. Sarzani R, Salvi F, Dessi-Fulgheri P, Rappelli A (2008) Renin-angiotensin system, natriuretic peptides, obesity,metabolic syndrome, and hypertension: An integrated view in humans. J Hypertens 26(5): 831843.

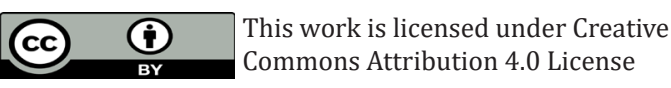

To Submit Your Article Click Here:

Submit Article

DOI: 10.32474/ADO.2018.01.000108

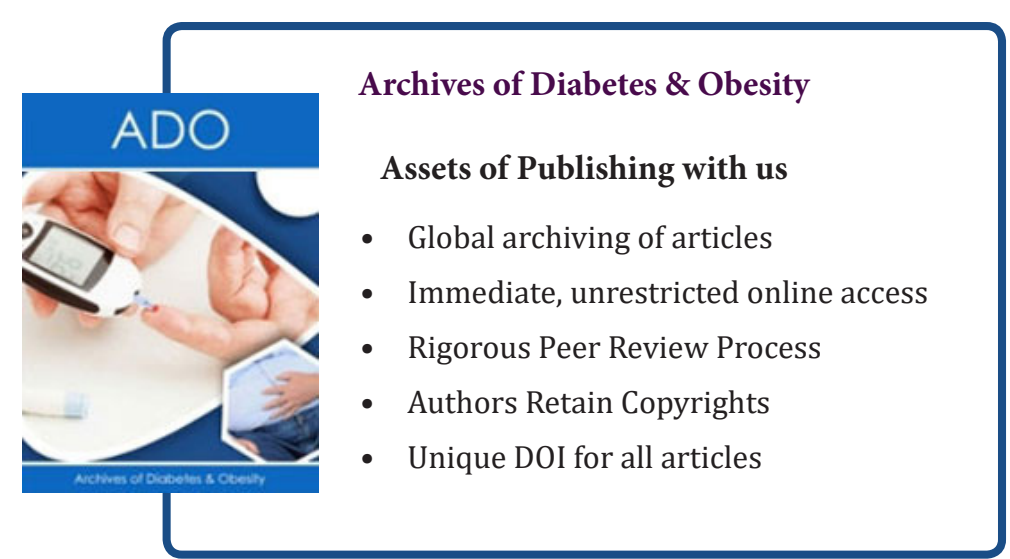

